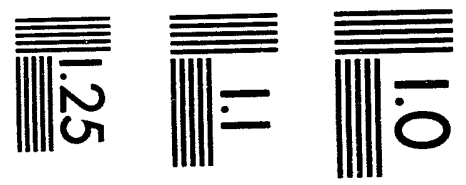

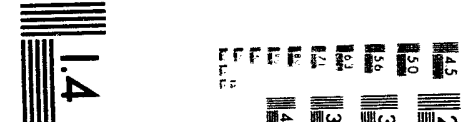

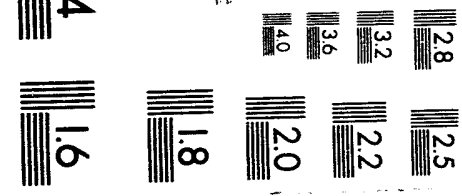


$-$
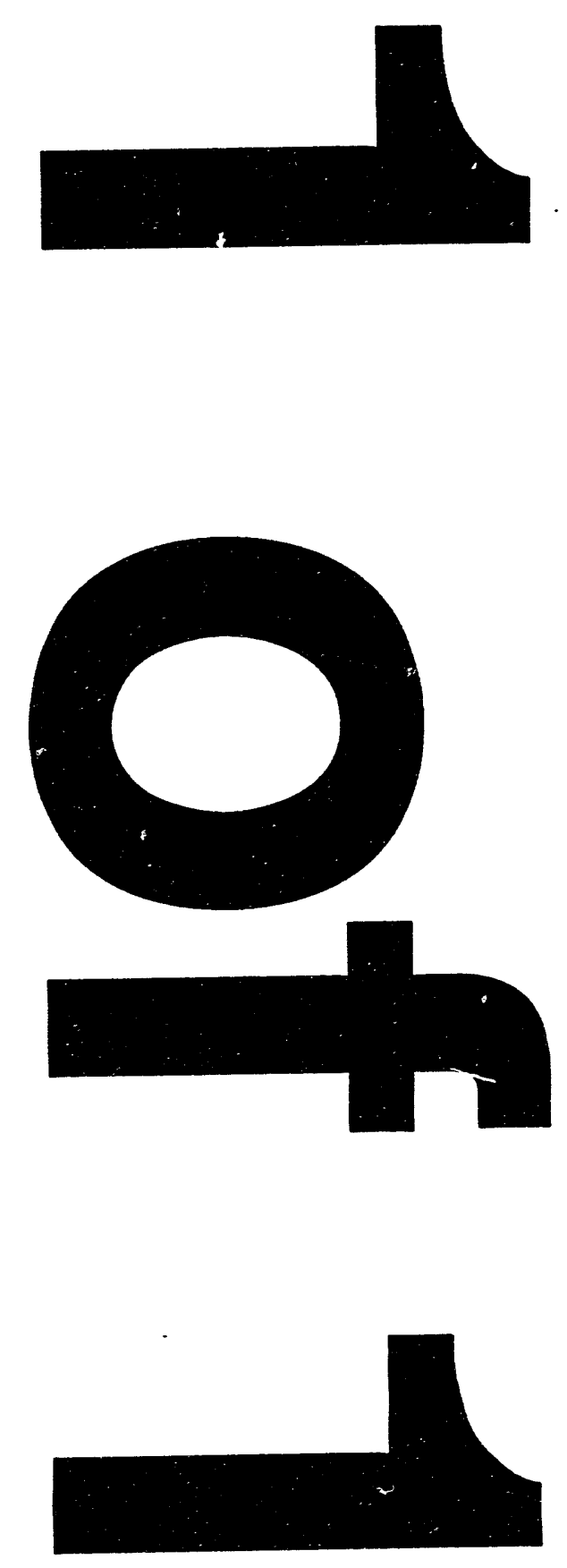


\section{HIGGS EFFECTS IN THE POLARIZATION OF TOP QUARKS}

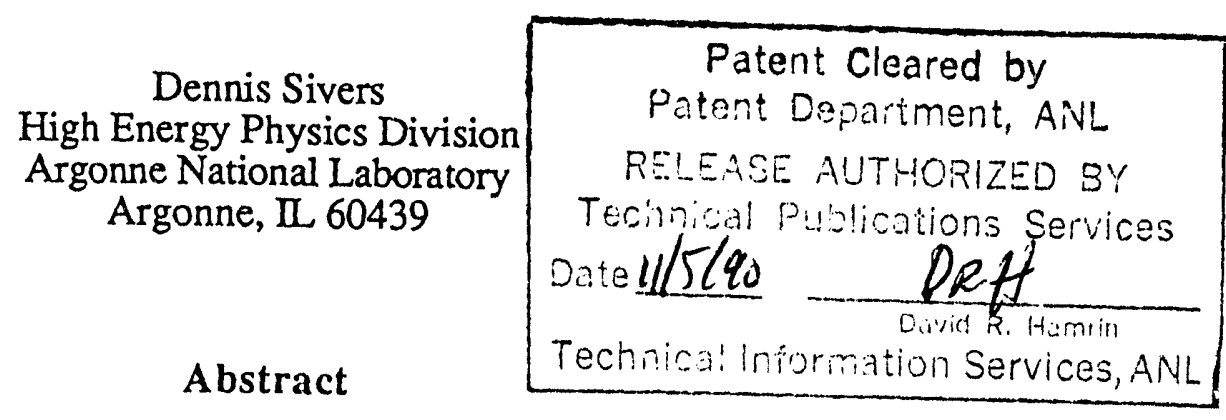

Top quarks produced in high-energy collisions should exhibit a parity-conserving spin polarization normal to the production plane which can be measured by the asymmetry in their leptonic decays. A portion of this effect, which can be calculated in perturbation theory, is attributable to the quark's QCD interactions. In addition, there is a component of the polarization due to the Yukawa coupling $\left(g_{Y} \cong m_{T} /(250 \mathrm{GeV})\right)$ of the top quark with the Higgs sector. We can demonstrate the interplay of QCD and Yukawa forces in the polarization for $e^{+} e^{-} \rightarrow T \bar{T}$. Assuming $M_{H}>2 M_{T}$ and that the Higgs particle is not discovered before the top quark, measurement of top quark polarization in $e^{+} e^{-}$or hadron-hadron collisions can be used to specify an approximate mass for the Higgs and guide direct searches. *Work supported by the U.S. Department of Energy, Division of High Energy Physics, Contract
W-31-109-ENG-38. 
The top quark is needed to complete the third family of leptons in the standard model. Its large mass (the current experimental bound is $M_{T} \geq 91 \mathrm{GeV}^{1}$ ) can make experimental detection difficult. However, this large mass also means that the top quark can play an important role in providing a gateway into the Higgs sector of the standard model or its variations. The current understanding of electroweak symmetry breaking requires a Yukawa coupling.

$$
g_{Y}^{H i i} \cong M_{i} /(250 G e V) \text {, }
$$

for the Higgs and any massive fermion. The existing spectrum of fermion masses ensures that the top quark dominates the hierarchy of these couplings.

In order to understand the nature of top quark interactions, we have to take into account the fact that for $M_{T} \geq 100 \mathrm{GeV}$, the Yukawa coupling for the Higgs- $T \bar{T}$ vertex, $g_{Y}$, is comparable in magnitude to the QCD coupling, $g_{s} / \sqrt{2}$, for the gluon-T $\bar{T}$ vertex. Regardless of whether the Higgs is an elementary scalar particle or a composite object, the scalar forces comesponding to boson exchange between top quarks are large and there should be experimental consequences of these forces. An obvious way to study these forces is to measure experimental quantities in which the amplitude for Higgs exchange interferes with the amplitude for a known production process. An example of such an observable is the transverse polarization of top quarks produced in high energy collisions. The parity-conserving polarization of a massive spin-1/2 particle defined as the expectation of the spin operator transverse to the production plane can be written ${ }^{2}$ in the helicity basis

$$
\begin{aligned}
\left\langle\sigma_{y}\right\rangle I(\theta) & =\sum_{\substack{\left(\lambda_{i}\right)\left(\lambda_{f}\right) \\
h_{T} \cdot h_{T}}} \sigma_{h_{T} h_{T}^{\prime}}^{y}\left\langle\left\{\lambda_{i}\right\}|A| h_{T}\left\{\lambda_{f}\right\}\right\rangle\left\langle\left\{\lambda_{i}\right\}|A| h_{T}^{\prime} ; \lambda_{f}\right\rangle \\
& =2 i \operatorname{Im} \sum_{\left\{\lambda_{i}\right)\left(\lambda_{F}\right\}}\left\langle\{\lambda\}|A|+; \lambda_{f}\right\rangle^{*}\left\langle\left\{\lambda_{i}\right\}|A|-;\left\{\lambda_{f}\right\}\right\rangle
\end{aligned}
$$

where $\left\{\lambda_{i}\right\}$ are the initial and $\left\{\lambda_{f}\right\}$ the final helicities of the other particles in the process. At the Born level of perturbation theory, the different helicity amplitudes in Eq. (2) are all real. We can generate the leading contribution to the above bilinear by calculating the imaginary part of the amplitudes through one loop. Using the Cutkosky rules, this involves the physical discontinuities 
in the s channel. For $e^{+} e^{-} \rightarrow T \bar{T}$, the Feynman diagram which generates the imaginary part of a given helicity amplitude by one gluon exchange is shown in Fig. 1.a. We can identify the imaginary part using $2 \rightarrow 2$ unitarity

$$
\begin{gathered}
\operatorname{Im}\left\langle\lambda_{1} \lambda_{2}\left|A_{e^{+} e^{-} \rightarrow T \bar{T}}\right| \lambda_{3} \lambda_{4}\right\rangle=\frac{1}{64 \pi^{2} E} \sum_{n} p_{n} \int d \Omega_{n}\left\langle\lambda_{1} \lambda_{2}\left|A_{e^{+} e^{-} \rightarrow n}\right|\left\{\lambda_{n}\right\}\right\rangle^{*} \\
\left\langle\left\{\lambda_{n}\right\}\left|A_{n \rightarrow T T}\right| \lambda_{3} \lambda_{4}\right\rangle
\end{gathered}
$$

For our purposes, it is instructive to take partial wave amplitudes

$$
\left\langle\lambda_{1} \lambda_{2}\left|A^{J}(E)\right| \lambda_{3} \lambda_{4}\right\rangle=\frac{1}{32 \pi} \int_{-1}^{+1} d z\left\langle\lambda_{1} \lambda_{2}|A(E, z)| \lambda_{3} \lambda_{4}\right\rangle d_{\lambda \lambda^{\prime}}^{J}(z)
$$

where $z=\cos \theta, \lambda=\lambda_{1}-\lambda_{2}, \lambda^{\prime}=\lambda_{3}-\lambda_{4}$. For $e^{+} e^{-} \rightarrow T \bar{T}$ we need only consider $J=1$ and the unitarity constraint gives

$$
\begin{aligned}
& \operatorname{Im}\left\langle\lambda_{1} \lambda_{2}\left|A_{e^{+} e^{-} \rightarrow T \bar{T}}^{J=1}\right| \lambda_{3} \lambda_{4}\right\rangle=\sum_{n=q \bar{q}}\left(\frac{p_{n}}{E}\right)\left\langle\lambda_{1} \lambda_{2}\left|A_{e^{+} e^{-} \rightarrow q \bar{q}}^{J=1}\right| \lambda_{5} \lambda_{6}\right\rangle^{*} \\
& \left\langle\lambda_{5} \lambda_{6}\left|A_{q \bar{q}}^{J=1} \rightarrow T T\right| \lambda_{4} \lambda_{5}\right\rangle
\end{aligned}
$$

To leading order in perturbation theory, we need only consider Born terms in the unitarity sum and we can write

$$
\begin{gathered}
P I(z)=\left(\frac{4}{E}\right) \sum_{\lambda_{i}, n} p_{n}\left\langle\lambda_{1} \lambda_{2}\left|A_{e^{+} e^{-} \rightarrow q \bar{q}}^{J=1}\right| \lambda_{5} \lambda_{6}\right\rangle\left\langle\lambda_{1} \lambda_{2}\left|A_{e^{+} e^{-} \rightarrow T \bar{T}}^{J=1}\right|-1 / 2, \lambda_{4}\right\rangle \\
\left\langle\lambda_{5} \lambda_{6}\left|A_{q \bar{q} \rightarrow T \bar{T}}^{J=1}\right|+1 / 2, \lambda_{4}\right\rangle d_{1}^{\prime \prime \lambda_{i}+1 / 2}(z) d_{1}^{\lambda \lambda_{4}-1 / 2}(z) \\
I(z)=\frac{d \sigma}{d \Omega}=\frac{1}{E^{2}} \sum_{\lambda_{i}} \mid\left\langle\lambda_{1} \lambda_{2}\right| A_{e^{+} e^{-\rightarrow T T}}^{J=1}\left\langle\langle \lambda _ { 3 } \lambda _ { 4 } \rangle | ^ { 2 } \left(\left. d_{1}^{\lambda \lambda^{\prime}}(z)\right|^{2} .\right.\right.
\end{gathered}
$$

with $p_{n}$ the momentum in the intermediate state. The polarization can be seen to be linear in the amplitude for $q \bar{q} \rightarrow T \bar{T}$. The QCD contribution to the sum in (6) involves only the $T \bar{T} \rightarrow T \bar{T}$ channel since all other amplitudes have an orthogonal color structure.

The QCD-generated polarization,

$$
P_{Q C D}=-\frac{1}{3} \alpha_{s}\left(1-M_{T}^{2} / E^{2}\right)^{-1 / 2}\left(\frac{M_{T}}{E}\right)\left(1-z^{2}\right)^{1 / 2} \frac{\left[2 z+4 M_{T}^{2} / E^{2}\right]}{\left[1+z^{2}+\left(M_{T} / E\right)^{2}\left(1-z^{2}\right)\right]}
$$


is associated with the one-gluon exchange diagram in the t-channel for $T \bar{T} \rightarrow T \bar{T}$. The transverse polarization can, in principle, be measured by observing the angular asymmetry in the leptonic decay of the top quark. ${ }^{4}$ It is important to realize that, because of the large mass of the top, this decay can be considered at the quark level and it is not necessary to worry about spin dilution effects due to the hadronization of the top quark into spinless mesons.

In addition to the QCD forces, the large Yukawa coupling for the top requires that one consider the second diagram in Fig. 1 . The $J=1$ partial wave helicity amplitudes from elementary Higgs exchange in the $T \bar{T}$ channel are given in Table 1 . The contribution from an s-channel Higgs pole vanishes when projected on $J=1$ and we will not include it here. The polarization attributable to the Higgs-gererated forces is then

$$
P_{H}=\frac{1}{4} \alpha_{Y}\left(1-M_{T}^{2} / E^{2}\right)^{1 / 2}\left(\frac{M_{T}}{E}\right)\left(1-z^{2}\right)^{1 / 2} A\left(z ; E, M_{T}, M_{H}\right)
$$

with $\alpha_{Y}=g_{Y}^{2} / 4 \pi$ and $A\left(z ; E, M_{T}, M_{H}\right)$ given in items of the functions in the table.

$$
A\left(z ; E, M_{T}, M_{H}\right)=\frac{z\left(L_{4}-L_{3}+\left(M_{T} / E\right) L_{2}-\left(M_{T} / E\right)^{2} L_{5}\right)-\left(M_{T} / E\right)^{2} L_{1}}{\left[1+z^{2}+\left(M_{T} / E\right)^{2}\left(1-z^{2}\right)\right]}
$$

For illustration, we have plotted the polarization $P_{H}$ for $M_{T}=175 \mathrm{GeV}, \quad E=250 \mathrm{GeV}$ and $M_{H}=1000 \mathrm{GeV}$ in Fig. 2. The equivalence of the scalar degrees of freedom of the massive gauge Bosons in the standard model ${ }^{5}$ allows the exchange of pseudoscalar bosons as indicated in the third diagram of Fig. 1. This diagram contributes both to the $T \bar{T} \rightarrow T \bar{T}$ ( channel ( $\Pi_{0}$-exchange) and to the $B \bar{B} \rightarrow T \bar{T}$ channel ( $\Pi_{+}$-exchange) in Eq. (6). The $J=1$ partial wave amplitudes for $\Pi_{o}$-exchange in $T \bar{T} \rightarrow T \bar{T}$ are given in Table 2 . Including pseudoscalar-exchange forces produces a component of polarization, $P_{\Pi}$, with

$$
P_{\Pi}=\frac{1}{4} \alpha_{Y}\left(1-M_{T}^{2} / E^{2}\right)^{1 / 2}\left(\frac{M_{T}}{E}\right)\left(1-z^{2}\right)^{1 / 2} B\left(z, E, M_{T}, M_{Z}\right)
$$

with

$$
B\left(z, E, M_{T}, M_{z}\right)=\frac{z\left(J_{4}+J_{3}\right)}{\left[1+z^{2}+\left(M_{T} / E\right)^{2}\left(1-z^{2}\right)\right]}
$$


Just as in the case of the Higgs, those portions of the amplitude which correspond to a pseudoscalar particle in the s-channel are orthogonal to the photon in $e^{+} e^{-} \rightarrow q \bar{q}$ and will not contribute to the imaginary part.

We have dwelt here, for simplicity, on the polarization of top quarks in $e^{+} e^{-} \rightarrow T \bar{T}$. We can approach the same issue for top quarks produced in hadron-hadron collisions. The large mass of the top lends credence to the application of the QCD-based parton model and suggests the calculation of polarization in the subprocesses $q \bar{q} \rightarrow T \bar{T}$ and $G G \rightarrow T \bar{T} \quad$ We will not examine these processes in detail except to I vie that the QCD-induced polarization involves many more channels in the unitarity sum, ${ }^{6}$ while $P_{H}$ and $P_{\pi}$ are the same as in $e^{+} e^{-} \rightarrow T \bar{T}$. The $G G \rightarrow T \bar{T}$ process allows for a possible contribution of an s-channel Higgs pole which can be very important for energies near the Higgs mass.

There have been numerous studies devoted to the subject of detecting the Higgs or exploring the consequences of its absence. ${ }^{7}$ We will noi attempt to summarize them here. There exists, however, a strong possibility of discovering the top quark and collecting a sample of several hundred top decays before definitive Higgs searches can explore the full range of allowable masses. In this case, the information gleaned from top quark polarization can be very helpful in estimating the Higgs mass. The Yukawa couplings of the Higgs sector offer a set of tools which deserve to be used fully. A phenomenological study of the implications of top quark polarizations is in progress.

\section{DISCLAIMER}

This report was prepared as an account of work sponsored by an agency of the United States Government. Neither the United States Government nor any agency thereof, nor any of their employees, makes any warranty, express or implied, or assumes any legal liability or responsibility for the accuracy, completeness, or usefulness of any information, apparatus, product, or process disclosed, or represents that its use would not infringe privately owned rights. Reference herein to any specific commercial product, process, or service by trade name, trademark, manufacturer, or otherwise does not necessarily constitute or imply its endorsement, recommendation, or favoring by the United States Government or any agency thereof. The views and opinions of authors expressed herein do not necessarily state or reflect those of the United States Government or any agency thereof. 
Table I

Partial Wave Amplitudes for $T \bar{T} \rightarrow T \bar{T}$ via Higgs Exchange

$$
N=\frac{g_{Y}^{2}}{32 \pi}\left(\delta_{i j} \delta_{l k}\right)_{c o l o r}
$$

\begin{tabular}{c}
$\gamma_{1} \gamma_{2} / \gamma_{3} \gamma_{4}$ \\
\cline { 2 - 4 }++ \\
\cline { 2 - 4 }+-
\end{tabular}

$L_{1}\left(E, M_{T}^{2}, M_{H}^{2}\right)=\frac{-2\left[\left(1-M_{T}^{2} / E^{2}\right)\left(1-M_{T}^{2} / E^{2}+M_{H}^{2} / 2 E^{2}\right)+\left(M_{H}^{2} / 2 E^{2}\right)\left(1-M_{T}^{2} / E^{2}+M_{H}^{2} / E^{2}\right) L\right]}{\left(1-M_{T}^{2} / E^{2}\right)^{3}}$

$L_{2}\left(E, M_{T}^{2}, M_{H}^{2}\right)=\frac{4\left(1-M_{T}^{2} / E^{2}\right)\left(1-M_{T}^{2} / E^{2}+M_{H}^{2} / 2 E^{2}\right)-2\left(1-M_{T}^{2} / E^{2}+M_{T}^{2}+M_{H}^{2} / 2 E^{2}\right)\left(1-M_{T}^{2} / E^{2}+M_{H}^{2} / 4 E^{2}\right) L}{\left(1-M_{T}^{2} / E^{2}\right)^{3}}$

$L_{3}\left(E, M_{T}^{2}, M_{H}^{2}\right)=\frac{-\left(1-M_{T}^{2} / E^{2}\right)\left(1-M_{T}^{2} / E^{2}-M_{H}^{2} / 2 E^{2}\right)-1 / 2\left(M_{H}^{2} / 2 E^{2}\right)^{2} L}{\left(1-M_{T}^{2} / E^{2}\right)^{3}}$

$L_{4}\left(E, M_{T}^{2}, M_{H}^{2}\right)=\left(\frac{M_{H}^{2}}{2 E^{2}}\right) \frac{2-\left(1-M_{T}^{2} / E^{2}+M_{H}^{2} / 2 E^{2}\right) L}{\left(1-M_{T}^{2} / E^{2}\right)^{2}}$

$L_{5}\left(E, M_{T}^{2}, M_{H}^{2}\right)=\frac{3\left(1-M_{T}^{2} / E^{2}\right)\left(1-M_{T}^{2} / E^{2}+M_{H}^{2} 2 E^{2}\right)-2\left(1-M_{T}^{2} / E^{2}+M_{H}^{2} / 4 E^{2}\right)^{2} L}{\left(1-M_{T}^{2} / E^{2}\right)^{3}}$

with $L=\ln \frac{\left(1+2 E^{2}-4 M_{T}^{2}\right)}{M_{H}^{2}}$ 
Table II

Partial Wave Amplitudes for $T \bar{T} \rightarrow T \bar{T}$ via Pseudoscalar Exchange

$$
N_{\Pi}=\frac{g_{Y}^{2}}{32 \pi}\left(\delta_{i j} \delta_{l k}\right)_{c o l o r}
$$

\begin{tabular}{c|cccc}
$\gamma_{1} \gamma_{2} / \gamma_{3} \gamma_{4}$ & ++ & +- & -+ & $\ddots$ \\
++ & & & & - \\
\cline { 3 - 5 }+- & 0 & 0 & 0 & $J_{4}$ \\
+ & 0 & 0 & $-J_{3}$ & 0 \\
-- & 0 & $-J_{3}$ & 0 & 0 \\
$J_{4}$ & 0 & 0 & 0 \\
\hline
\end{tabular}

$$
\begin{aligned}
& J_{3}\left(E, M_{T}^{2}, M_{Z}^{2}\right)=-1+\frac{M_{z}^{2}}{2\left(E^{2}-M_{T}^{2}\right.}+\frac{1}{2}\left(\frac{M_{z}^{2}}{2\left(E^{2}-M_{T}^{2}\right)}\right)^{2} \ln \left(1+4\left(E^{2}-M_{T}^{2}\right) / M_{z}^{2}\right) \\
& J_{4}\left(E, M_{T}^{2}, M_{Z}^{2}\right)=-\frac{1}{2}\left(\frac{M_{Z}^{2}}{E^{2}-M_{T}^{2}}\right) \ln \left(1+4\left(E^{2}-M_{T}^{2}\right) / M_{Z}^{2}\right)
\end{aligned}
$$




\section{Acknowledgments}

I am very grateful for guidance by $C$. Zachos aild for important conversations on the equivalence theorem by C. P. Yuan.

\section{References}

1. CDF Collaioration.

2. M. Jacob and G. C. Wick, Annals of Physics (NY) 7, 404 (1959).

3. R. E. Cutkosky, J. Math Phys. 1, 429 (1960).

4. J. Kühn, Nuclear Physics B237, 77 (1984).

5. B. W. Lee, C. Quigg, and G. B. Thacker, Phys. Rev. Lett. 38, 883 (1977); Phys. Rev. D16, 1519 (1977). M. Chanowitz and M. K. Gaillard, Phys. Lett. 142B, 85 (1984) Nucl. Phys. B261, 379 (1085).

6. W.G.D. Dharmaratna, G. R. Goldstein and G. A. Ringland, Z. Phys. C.41, 673 (1988). W.G.D. Dharmaratna and G. R. Goldstein, Phys. Rev. D41, 1731 (1990).

7. See, for example, J. F. Gunion, H. E. Halser, G. Kane, and S. Dawson, The Higgs

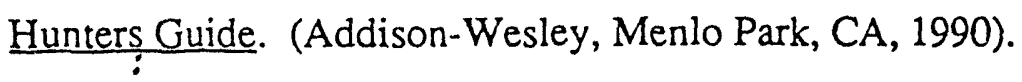




\section{Figure Captions}

Fig. 1. The diagrams which contribute to the imaginary parts of the helicity amplitudes for $e^{+} e^{-} \rightarrow T \bar{T}$. Diagram (a) shows gluon exchange in $T \bar{T} \rightarrow T \bar{T}$. Diagram (b) shows scalar Higgs exchange while (c) shows the exchange of a Pseudoscalar representing the longitudinal $z$.

Fig. 2. Top quark polarization as generated by Higgs exchange for $M_{T}=175 \mathrm{GeV}$, $E=250 \mathrm{GeV}$ and $M_{H}=1000 \mathrm{GeV}$. For these parameters $P_{H}$ dominates $P_{Q C D}$ and $P_{\pi}$. For $M_{H}^{2}>M_{T}^{2}$ the polarization is proportional to $M_{H}^{2}$ and this means that an experiment to measure $P$ can determine $M_{H}$. 


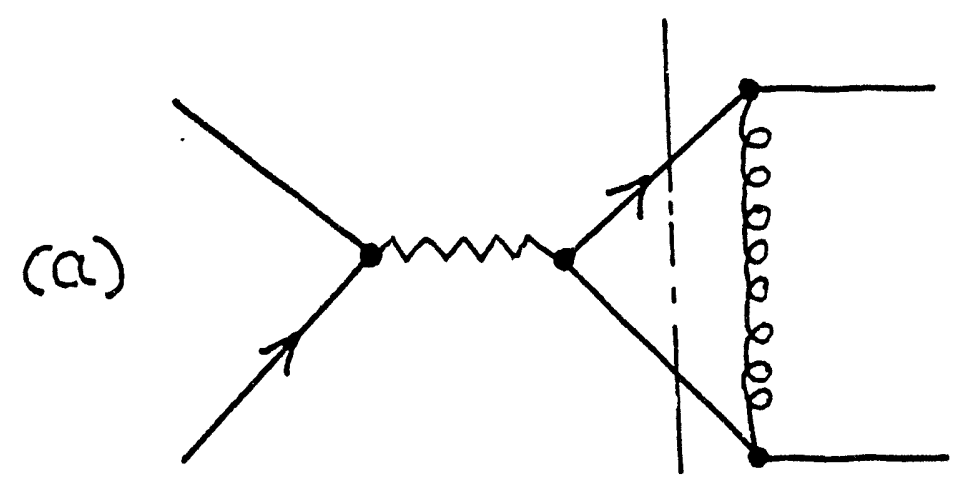

GLUON

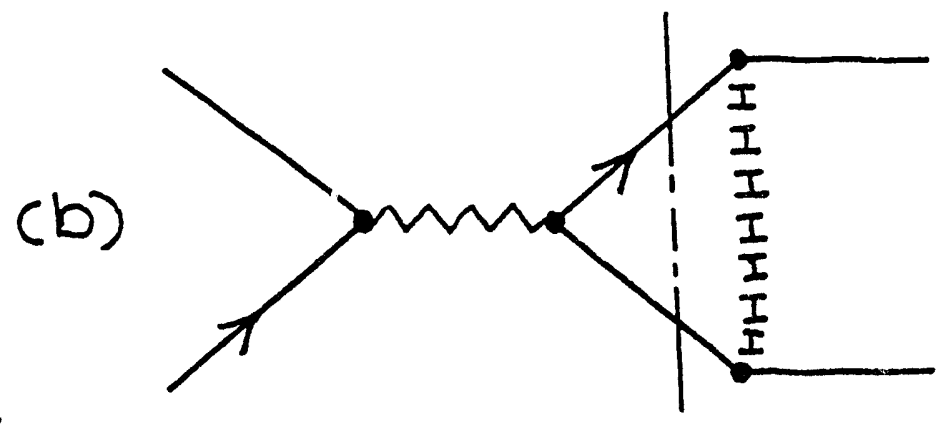

HIGES

(c)

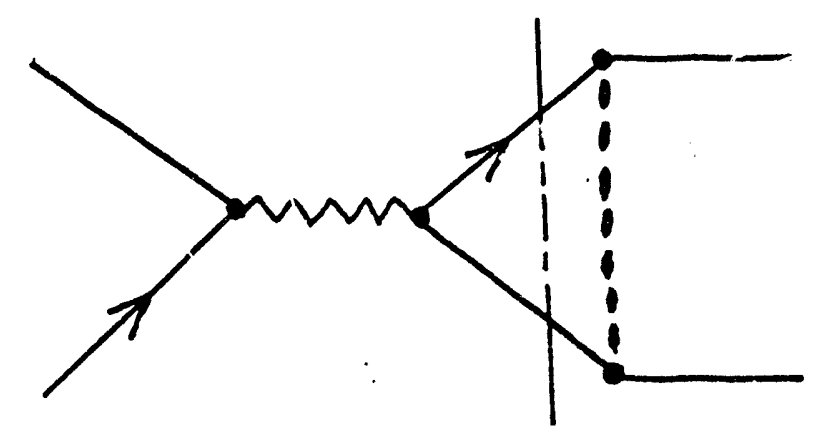

"ז" 


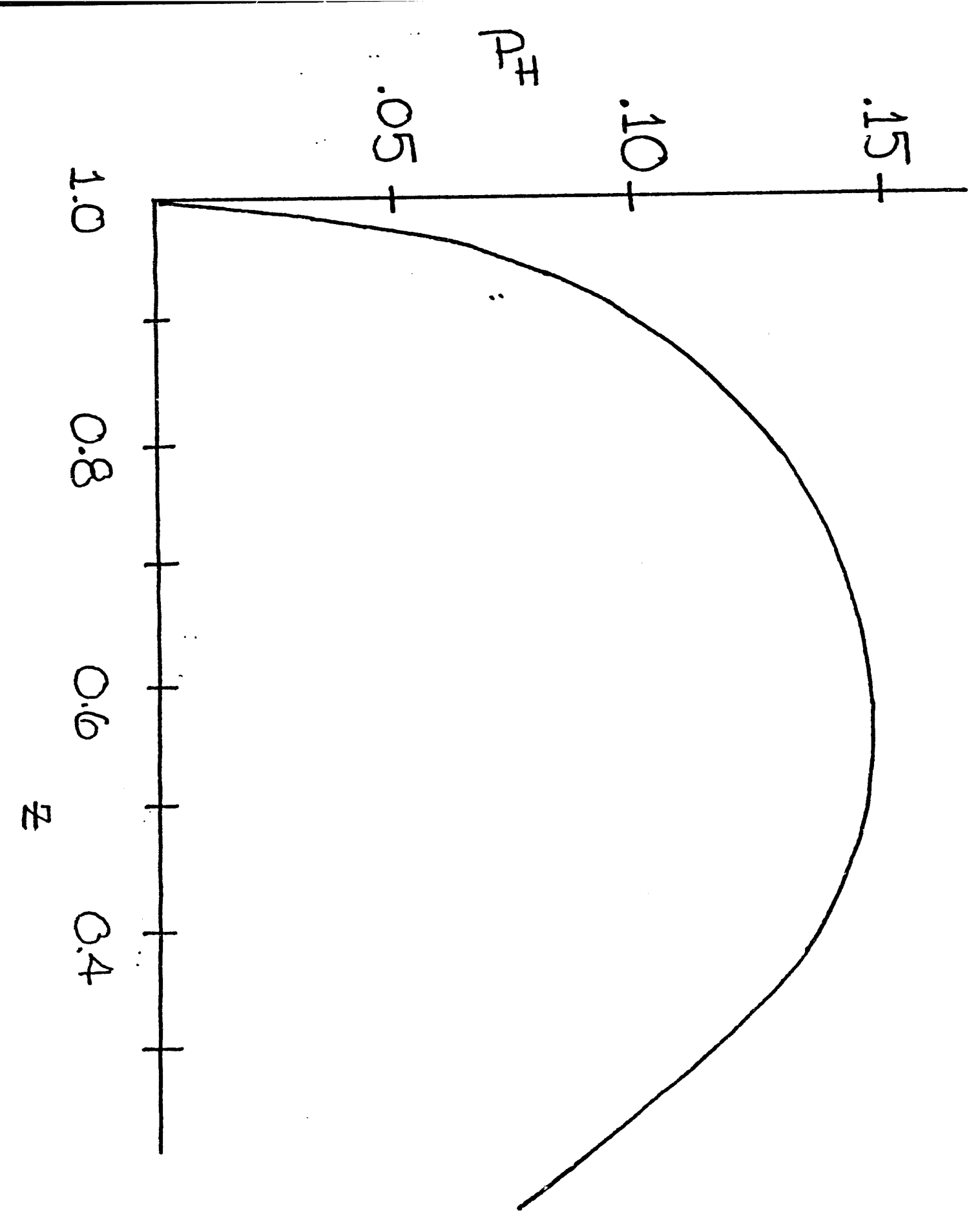

. 

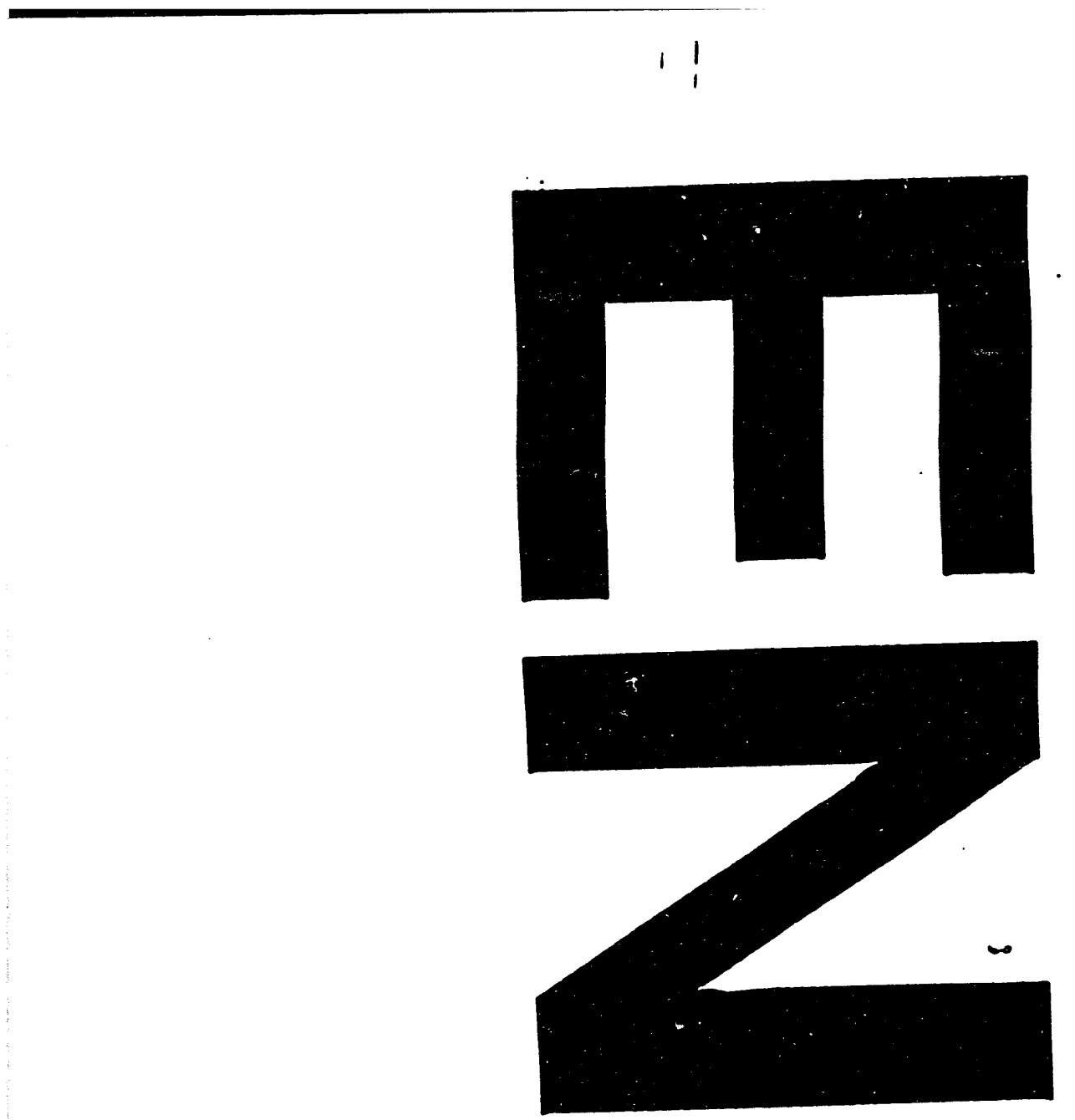

G
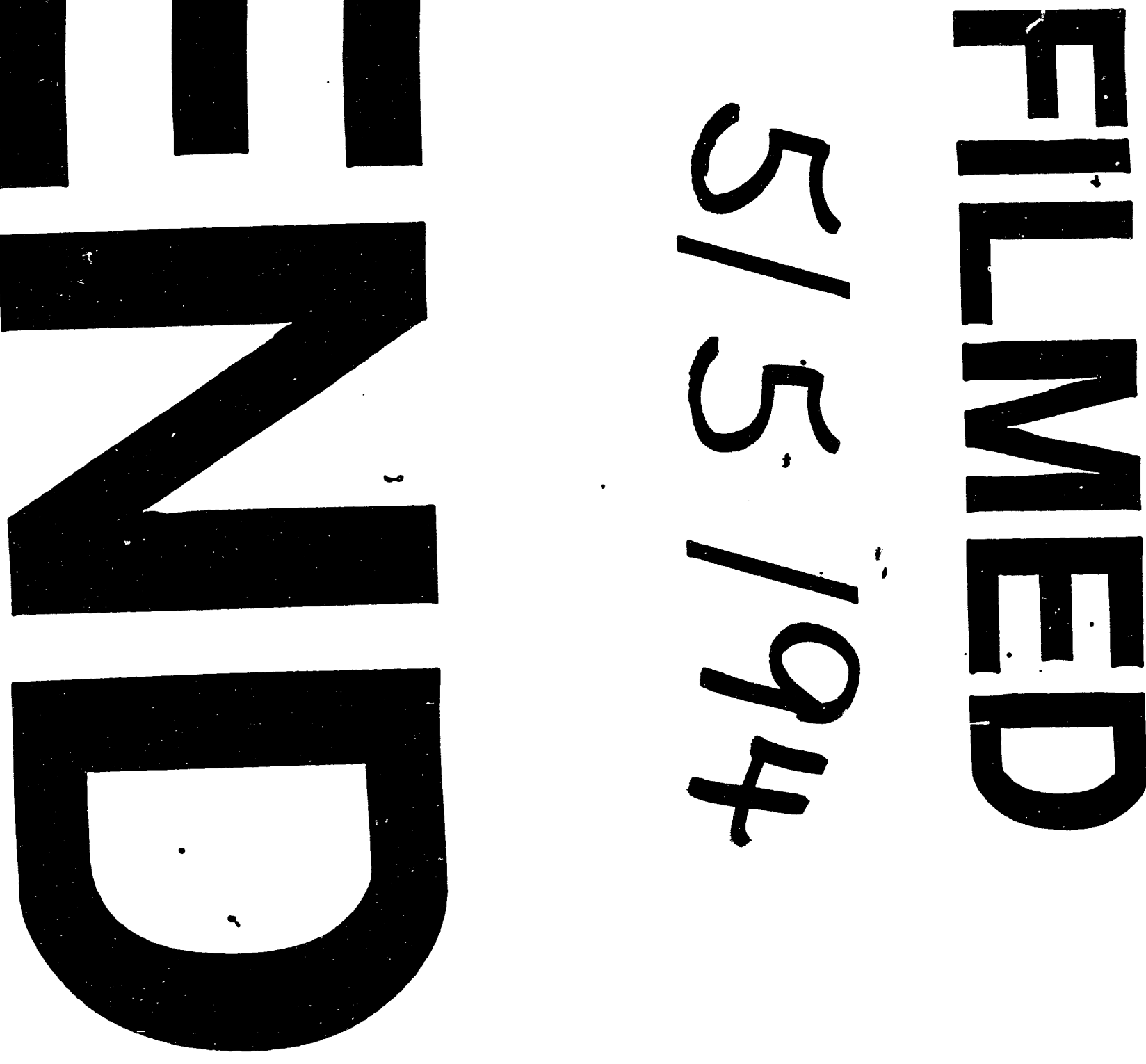

G
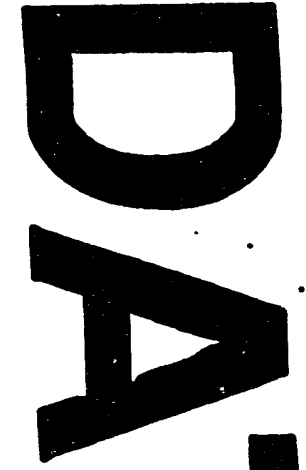

$\rightarrow$

$-$ $F$

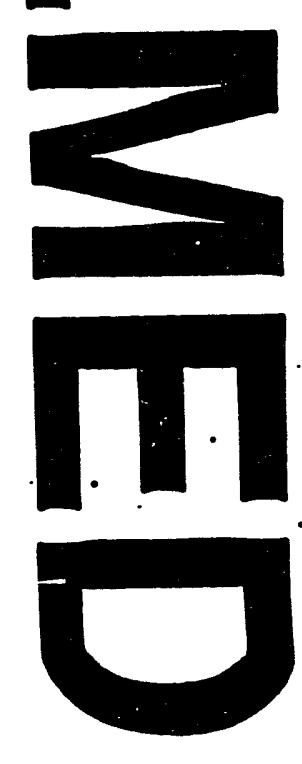


